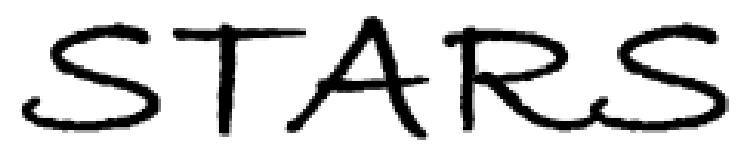

University of Central Florida

STARS

$1-1-2008$

\title{
Interband optical pulse injection locking of quantum dot mode- locked semiconductor laser
}

Jimyung Kim

University of Central Florida

Peter J. Delfyett

University of Central Florida

Find similar works at: https://stars.library.ucf.edu/facultybib2000 University of Central Florida Libraries http://library.ucf.edu

This Article is brought to you for free and open access by the Faculty Bibliography at STARS. It has been accepted for inclusion in Faculty Bibliography 2000 s by an authorized administrator of STARS. For more information, please contact STARS@ucf.edu.

\section{Recommended Citation}

Kim, Jimyung and Delfyett, Peter J., "Interband optical pulse injection locking of quantum dot modelocked semiconductor laser" (2008). Faculty Bibliography 2000s. 545.

https://stars.library.ucf.edu/facultybib2000/545

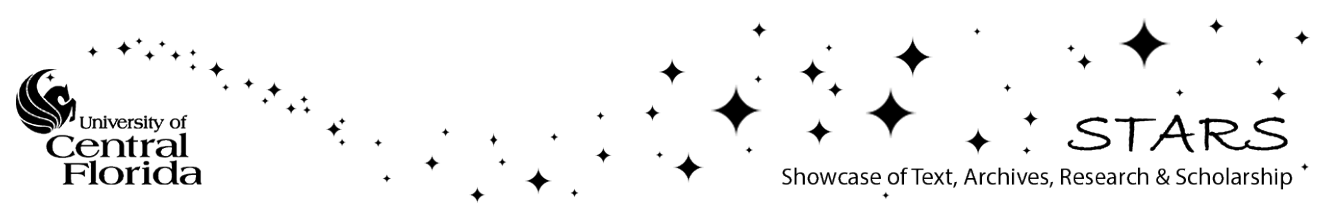




\title{
Interband optical pulse injection locking of quantum dot mode-locked semiconductor laser
}

\author{
Jimyung Kim and Peter J. Delfyett \\ CREOL, The College of Optics and Photonics, University of Central Florida, Orlando, FL 32816, USA \\ jmkim@creol.ucf.edu delfyett@creol.ucf.edu
}

\begin{abstract}
We experimentally demonstrate optical clock recovery from quantum dot mode-locked semiconductor lasers by interband optical pulse injection locking. The passively mode-locked slave laser oscillating on the ground state or the first excited state transition is locked through the injection of optical pulses generated via the opposite transition bands, i.e. the first excited state or the ground state transition from the hybridly modelocked master laser, respectively. When an optical pulse train generated via the first excited state from the master laser is injected to the slave laser oscillating via ground state, the slave laser shows an asymmetric locking bandwidth around the nominal repetition rate of the slave laser. In the reverse injection case of, i.e. the ground state (master laser) to the first excited state (slave laser), the slave laser does not lock even though both lasers oscillate at the same cavity frequency. In this case, the slave laser only locks to higher injection rates as compared to its own nominal repetition rate, and also shows a large locking bandwidth of $6.7 \mathrm{MHz}$.
\end{abstract}

(C)2008 Optical Society of America

OCIS codes: (140.5960) Semiconductor laser; (140.4050) Mode-locked laser; (140.3520) Laser, injection-locked; (250.5590) Quantum-well, -wire and -dot devices.

\section{References and links}

1. R. Lang and K. Koobayashi, "Suppression of the relaxation oscillation in the modulated output of semiconductor lasers," IEEE J. Quantum Electron. QE-12, 194-199 (1976).

2. A. Takada and W. Imajuku, "Linewidth narrowing and optical phase control of mode-locked semiconductor ring laser employing optical injection locking," J. Opt. Soc. Am. A. 14, 741-755 (1997).

3. M. Margalit, M. Orenstein, and H. A. Haus, "Injection locking of a passively mode-locked laser," IEEE J. Quantum Electron. vol. 32, 155-160 (1996).

4. B. K. Mathason and P. J. Delfyett, "Pulsed injection locking dynamics of passively mode-locked externalcavity semiconductor laser systems for all-optical clock recovery," IEEE J. Lightwave Technol. 18, 1111 $1120(2000)$

5. W. Lee and P. J. Delfyett, "Dual-mode injection locking of two independent modelocked semiconductor lasers," IEE Electron. Lett. 40, 1182-1183 (2004).

6. H. Kurita, T. Shimizu, and H. Yokoyama, "Experimental investigation of harmonic synchronization conditions and mechanisms of mode-locked laser diodes induced by optical-pulse injection," IEEE J. Sel. Top Quantum Electron. 2, 508-513 (1996).

7. S. Arahira and Y. Ogawa, "Synchronous mode-locking of passively mode-locked semiconductor laser diodes by using optical short pulses repeated at subharmonics of the cavity roundtrip frequencies," IEEE. Photon. Technol. Lett. 8, 191-193 (1996)

8. D. Bimberg, N. Kirstaedter, N. N. Ledentsov, Zh. I. Alferov, P. S. Kop'ev, and V. M. Ustinov, "InGaAsGaAS Quantum-Dot Lasers," IEEE J. Sel. Top. Quantum Electron. 3, 196-205 (1997).

9. S. Schneider, P. Borri, W. Langbein, U. Woggon, R.L. Sellin, D. Ouyang, and D. Bimberg, "Excited-state gain dynamics in InGaAs quantum-dot amplifers," IEEE Photon. Technol. Lett. 13, 2014-2016 (2005).

10. J. Kim, M. T. Choi, and P. J. Delfyett "Pulse generation and compression via ground and excited states from a grating coupled passively mode-locked quantum dot two section diode laser," Appl. Phys. Lett. 89, 261106 (2006).

11. A. E. Zhukov, A. R. Kovsh, N. A. Maleev, S. S. Mikhrin, V. M. Ustinov, A. F. Tsatsul'nikov, M. V. Maximov, B. V. Volovik, D. A. Bedarev, Yu. M. Shernyakov, P. S. Kop'ev, Zh. I. Alferov, N. N. Ledentsov, and D. Bimberg, "Long-wavelength lasing from multiply stacked InAs/InGaAs quantu dots on GaAs substrates," Appl. Phys. Lett. 75, 1926 (1999). 
12. C. E. Zah, R. Bhat, S. G. Menocal, N. Andreadakis, F. Favire, C. Caneau, M. A. Koza, and T. P. Lee, "1.5 $\mu \mathrm{m}$ GaInAsP angled-facet flared-waveguide traveling-wave laser amplifiers,” IEEE Photon. Technol. Lett. 2, 46-47 (1990)

\section{Introduction.}

Injection locking of semiconductor lasers has been considered as an effective technique to control lasers both in the frequency and time domains. Reducing mode partition noise and relaxation oscillations, narrowing the linewidth and synchronizing many lasers to a single master laser using small injection powers have been demonstrated by injection locking techniques [1-5]. Clock recovery using injection locking techniques such as sub-harmonic, fundamental or harmonic optical pulse injection has been demonstrated and studied using quantum well (QW) [4-7]. Quantum dot (QD) based mode-locked lasers as a clock recovery source is now an attractive choice for high speed all-optical clock recovery due to its superior characteristics, such as its potential for low noise and fast gain recovery, as well as its broad spectrum $(\sim 100 \mathrm{~nm})$ which originates from the ground state (GS) and the excited state (ES) transitions [8-9]. Recently, the pulse characteristics of quantum dot based mode-locked lasers operating exclusively on the ground state and the excited state transitions have been studied for a two band clock recovery source for telecommunication application [10].

Until now, clock recovery using optical pulse injection locking techniques has been only achieved by sharing the same spectral band between the master and slave laser under injection locked conditions [4-7]. In this work, we have experimentally observed that optical pulse injection locking between a master and a slave laser is possible when a master and a slave laser oscillate via different states, e.g., using either GS or ES transitions. This interband optical pulse injection locking will overcome the physical consideration of sharing the same spectral band between master and slave lasers for injection locking. As a result, one master laser can control different slave lasers whose finite lasing spectral band does not overlap with that of the master laser.

\section{Experimental Schematic and Setup.}

For this experiment, a QD two-section device which has a gain and saturable absorber section, and a semiconductor optical amplifier (SOA), were fabricated with a ridge-waveguide structure from a QD wafer whose active region is formed by ten layers of InAs quantum dots covered by an InGaAs quantum well layer [11]. The length of gain and saturable absorber of the QD two-section device is $2 \mathrm{~mm}$ and $0.25 \mathrm{~mm}$, respectively. The gain section is curved with an angle of $7^{\circ}$ and tapered at termination to minimize the back reflection from the facet [12]. The QD SOA is also $7^{\circ}$ tilted and has a $3 \mathrm{~mm}$ length. No high-reflection or antireflection coating was applied onto these devices.

For interband optical pulse injection locking, two grating coupled quantum dot external lasers (master and slave) were constructed so each laser operated with a nominal pulse repetition frequency of $4 \mathrm{GHz}$. Hybrid mode-locking and passive mode-locking is performed on the master laser and the slave laser, respectively. Figure 1 shows the interband optical pulse injection locking setup. An optical pulse train generated from the master laser oscillating on the opposite transition (GS or ES) with respect to the slave laser (ES or GS) is injected into the saturable absorber of the slave laser. The injection power level is controlled by a neutral density filter (NDF). The output of the slave laser is taken from the saturable absorber section. The partially reflected output of the slave laser by the pellicle is passed through an optical bandpass filter and then is amplified through a QD SOA. Since the optical bandpass filter allows the spectral band of the slave laser to pass, only the spectral band of the interband optical pulse injected slave laser is detected and analyzed.

The interband optical pulse injection locking experiment has been done by considering two cases. In the first case, the pulses from ES transition of the master laser are injected to the slave laser oscillating via the GS transition. The second case examined pulses from the slave laser operating on the ES transition injected with pulses generated via GS transition from the master laser. The lasing band operation on the GS or ES transition is achieved through a 
grating (9001n/mm) which is externally coupled to a QD curved 2-section device in the Littrow configuration. Experimental details and results are discussed in the subsequent sections.

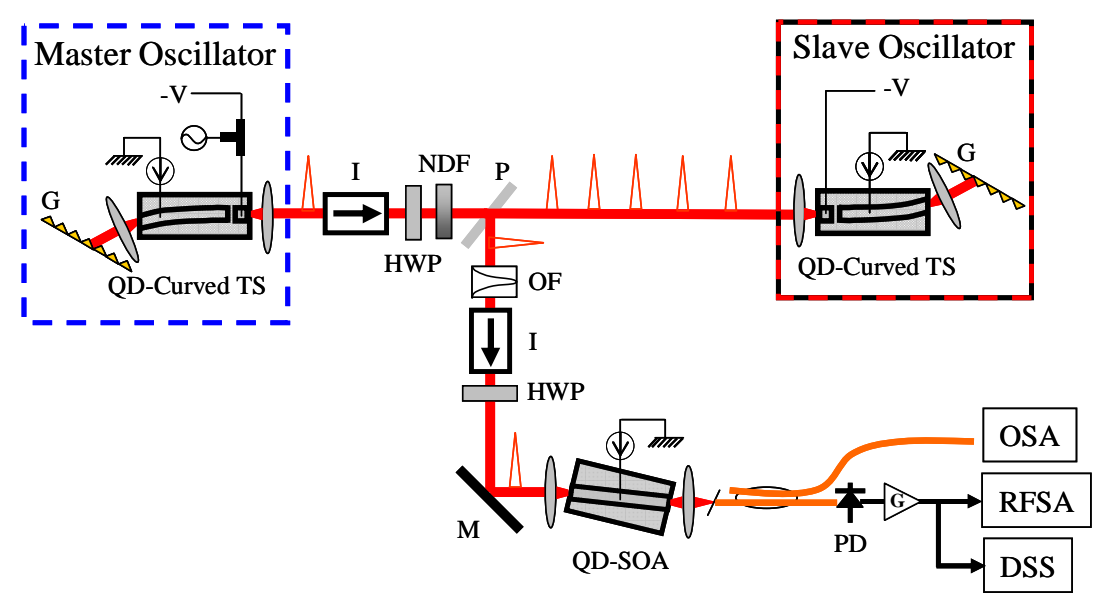

Fig. 1. Schematic diagram of interband optical pulse injection locking experiment. (QD-Curved TS: quantum dot curved two section diode, G: grating, I: isolator, NDF: neutral density filter, HWP: half wave plate, P: pellicle, OF: optical filter, M: mirror, QD SOA: quantum dot semiconductor optical amplifier, PD: photo detector, OSA: optical spectrum analyzer, RFSA: RF spectrum analyzer, DSS: digital sampling scope)

\section{ES optical pulse injection to the slave laser oscillating via GS transition}

First, the optical pulse train from the master laser operating on the ES transition is injected to the saturable absorber of the slave laser oscillating on the GS transition. The two bands (ES and GS) in the saturable absorber of the slave laser are bleached simultaneously by the injected ES optical pulses and the GS optical pulses from the hybridly mode-locked master laser and the passively mode-locked slave laser, respectively.

The bias current of $134 \mathrm{~mA}$ to the gain section, a reverse bias of $0 \mathrm{~V}$ and an RF power of $15 \mathrm{dBm}$ to the saturable absorber section enables the master laser to be hybridly mode-locked via the ES transition. The slave laser is passively mode-locked via the GS transition by applying a reverse bias of $5.7 \mathrm{~V}$ to the saturable absorber and a bias current of $37 \mathrm{~mA}$ to the gain section. Both mode-locked lasers nominally operate at the same $4 \mathrm{GHz}$ repetition rate. An angle tuning of the externally coupled gratings enables the master and slave laser to lase via the ES transition $(1186.5 \mathrm{~nm})$ and GS transition $(1267.5 \mathrm{~nm})$, respectively. The corresponding optical spectra are shown in Fig. 2. Lasing via the ES transition is achieved through ES amplified spontaneous emission (ASE) generated after a saturation of GS ASE.

To investigate the dynamics of the interband optical pulse injected slave laser, ES optical pulses are injected to the slave laser. The average power of the injected signal is $\sim 1 \mathrm{~mW}$. A long pass filter whose cut-on wavelength is $1250 \mathrm{~nm}$ is used as an optical filter. It filters the optical power of the master laser partially reflected from the facet of the slave laser, so the optical pulse train generated from the ES optical pulse injected slave laser oscillating via GS transition is only amplified through a QD SOA and is analyzed. 


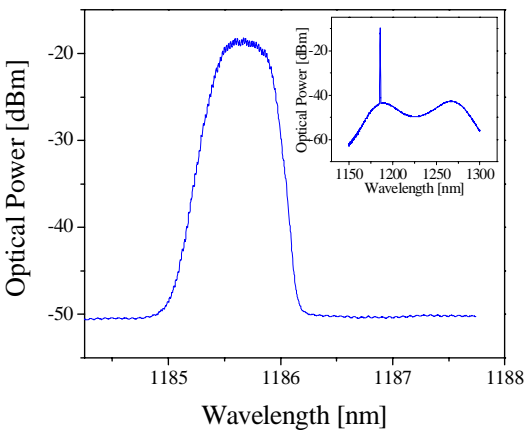

(a)

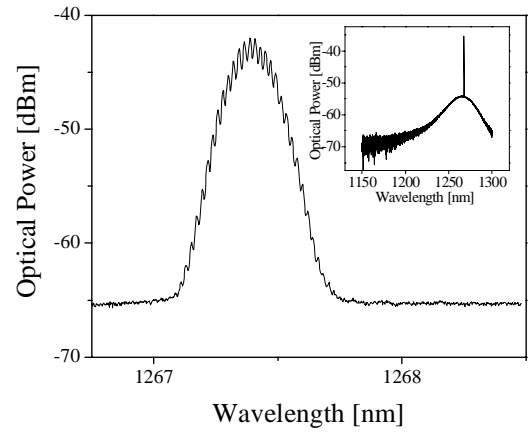

(b)

Fig. 2. (a). ES optical spectrum of the hybridly mode-locked master laser, inset: wide span optical spectrum and (b) GS optical spectrum of the passively mode-locked slave laser, inset: wide span optical spectrum.

The injected interband optical pulse doesn't induce any noticeable change in the optical spectrum of the slave laser as shown in Fig. 3(a). The resolution of the OSA is $0.01 \mathrm{~nm}$. It should be noted that the ES pulse train isn't supported in the slave diode itself due to an insufficient injected current level on the gain section of the slave laser and also due to the round trip losses experienced by the ES pulse due to the externally coupled grating which only reflects the GS optical pulse back to the slave diode laser. As a result, the injected ES optical pulse train first saturates the ES band in the saturable absorber of the slave laser and then is attenuated through the slave oscillator.

The phase and amplitude noise of the slave laser are reduced and an optical pulse train is observed after the injection of the interband optical pulses. As shown Figs. 3(b) and 3(c), the noise reduction and stable optical pulse train generation of the slave laser mean that the slave laser is locked to the master laser. The video and resolution bandwidth of RFSA are both 10 $\mathrm{kHz}$.

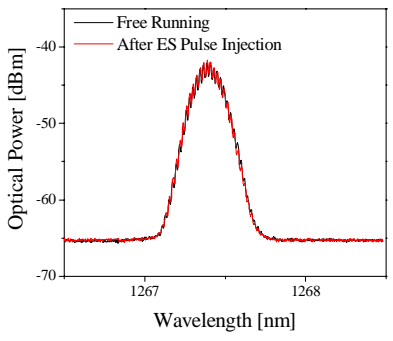

(a)

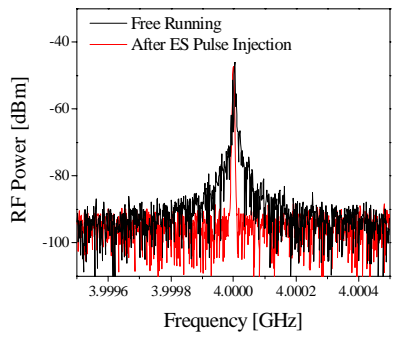

(b)

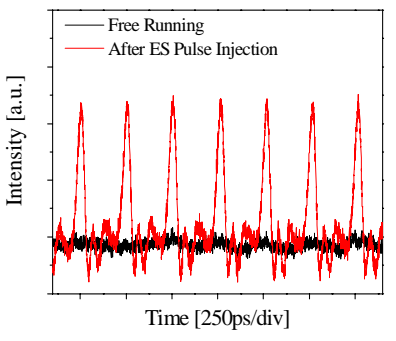

(c)

Fig. 3. (a). optical spectrum, (b) RF power spectrum and (c) digital sampling scope trace of the passively mode-locked slave laser oscillating via GS transition before and after ES optical pulse train injection.

We measure the locking bandwidth of the slave laser by changing the repetition rate of the master laser and varying the injection power. The pulse repetition rate of the master laser is achieved by changing the frequency of the signal generator used for hybrid mode-locking. The repetition rate of the master laser is detuned from $+500 \mathrm{kHz}$ to $-500 \mathrm{kHz}$ with a noise suppression of $70 \mathrm{~dB}$. The optical injection power level to slave laser is controlled by a NDF. Figure 4(a) shows the locking bandwidth in terms of the injected optical power versus input 
pulse repetition rate and Fig. 4(b) shows the RF spectrum of the slave laser at the maximum detuning of $500 \mathrm{kHz}$ with an estimated $1 \mathrm{~mW}$ of injection power. Locking is determined by observing a $>30 \mathrm{~dB}$ reduction of the sideband noise from the fundamental tone of RFSA and also by the optical pulse train shown from DSS.

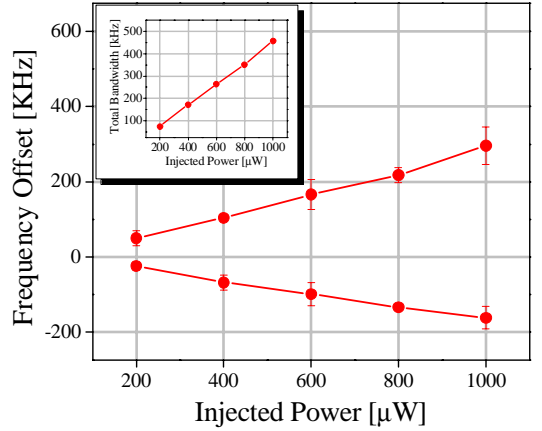

(a)

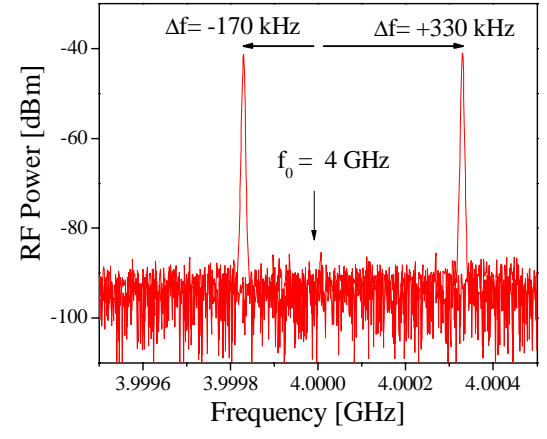

(b)

Fig. 4. (a). locking bandwidth and (b) RF spectrum of the interband optical pulse injected slave laser.

The slave laser is locked to much higher injection rate rather than lower injection rate compared to the nominal repetition rate at a given injected optical power. The asymmetric locking bandwidth range can be explained by the causality of the saturable absorber not by the carrier-induced refractive index change caused by the injected interband optical pulses. Since the increase of index induced by ES gain depletion will result in an increase of the effective cavity length of the slave laser and the slave laser would be locked to much lower injection rate compared to the nominal repetition rate of the laser [4].

\section{GS optical pulse injection to the slave laser oscillating via ES transition}

In the second experiment, a GS optical pulse train from the master laser is injected to the saturable absorber of the slave laser operating on the ES transition. Both the GS and ES bands in the saturable absorber of the slave laser are bleached at the same time by the injected GS optical pulse from the hybridly mode-locked master laser and the ES optical pulse from the passively mode-locked slave laser, respectively.

A bias current of $75 \mathrm{~mA}$ to the gain section, a reverse bias of $0 \mathrm{~V}$ and RF power of 15 $\mathrm{dBm}$ to the saturable absorber section are applied to the hybridly mode-locked GS master laser. For the operation of the slave laser via the ES transition, a bias current of $115 \mathrm{~mA}$ and a reverse bias of $1.5 \mathrm{~V}$ are applied to the gain and the saturable absorber section, respectively. Both lasers are mode-locked at fundamental cavity length of $4 \mathrm{GHz}$ and the lasing wavelengths are $1271 \mathrm{~nm}$ and $1191.8 \mathrm{~nm}$ for the master and slave laser, respectively as shown in Fig. 5. 


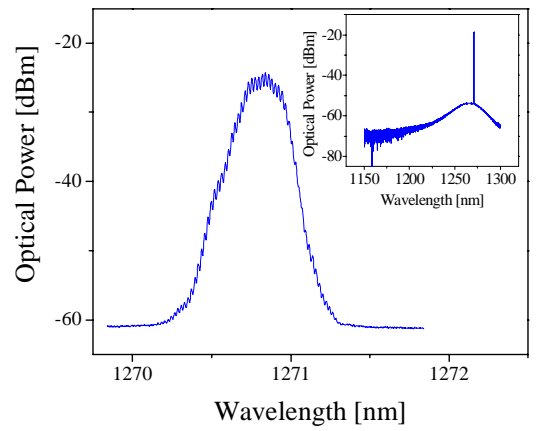

(a)

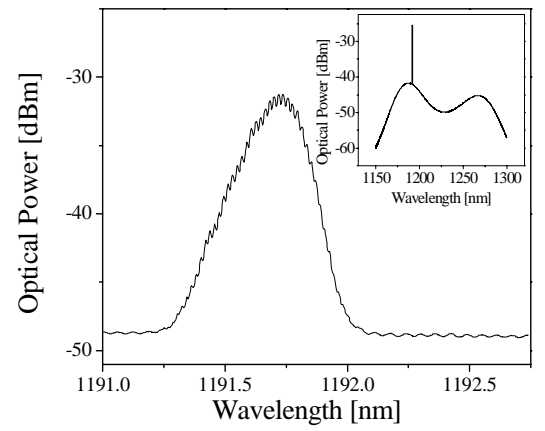

(b)

Fig. 5. (a). GS optical spectrum of the hybridly mode-locked master laser, inset: wide span optical spectrum and (b) ES optical spectrum of the passively mode-locked slave laser, inset: wide span optical spectrum.

To observe the dynamics of the interband injected slave laser oscillating via the ES transition, the GS optical pulses with an estimated average power of $1 \mathrm{~mW}$ are injected to the slave laser. It should be noted that the injected GS optical pulse can experience gain in the slave laser diode itself owing to the large bias current needed to achieve a population inversion on the ES transition. This large bias current also creates a population inversion at the GS transition. Nonetheless, the injected GS optical pulses are not resonant in the slave laser cavity due to the externally coupled grating which allows only the ES optical pulses to feedback into the slave laser diode. An optical bandpass filter with center wavelength of 1200 $\mathrm{nm}$ and a full width half maximum of $10 \mathrm{~nm}$ is inserted before QD SOA and the center wavelength of the optical bandpass filter is adjusted to $1192 \mathrm{~nm}$ by tilting the filter slightly. Since the filter blocks the GS optical pulse train partially reflected from the facet of the slave laser and only passes the ES optical band of the slave laser, we can investigate the dynamics of the GS optical pulse injected slave laser oscillating via ES transition.

When the ES optical pulse of $\sim 1 \mathrm{~mW}$ average power is injected to the slave laser oscillating through the GS transition, the slave laser does not lock even though the master and slave laser both oscillate at the same repetition rate of $4 \mathrm{GHz}$. The optical band of the slave laser is reduced and also distorted after injection of the interband optical pulses. Figure 6 shows the optical and RF spectrum of the slave laser before and after an injection of the GS optical pulses.

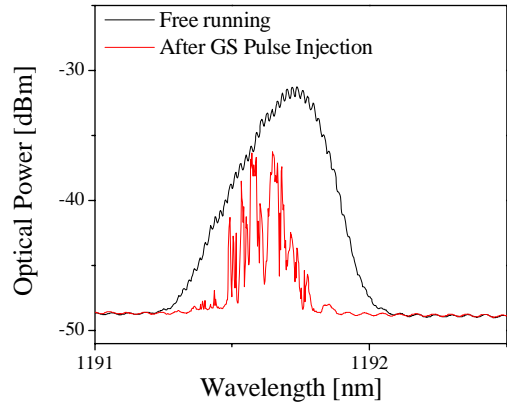

(a)

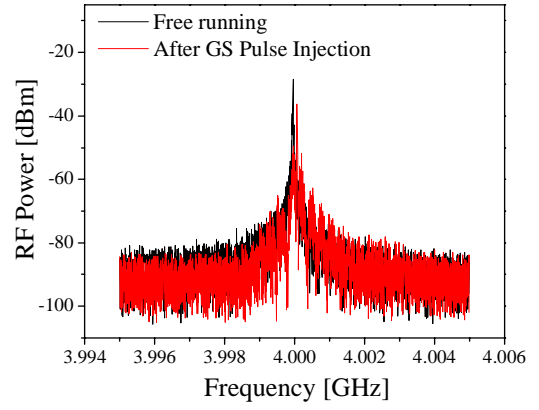

(b)

Fig. 6. (a). the optical spectrum and (b) RF spectrum of the slave laser oscillating via ES transition before and after GS optical pulse train injection.

\#93709 - \$15.00 USD

(C) 2008 OSA 
To investigate how the slave laser oscillating via ES transition responds to the different injection rate of GS optical pulses, the pulse repetition rate of the hybridly mode-locked master laser is detuned from $3.991 \mathrm{GHz}$ to $4.014 \mathrm{GHz}$ by the signal generator. The performance of the detuned master laser is shown in Fig. 7(a).

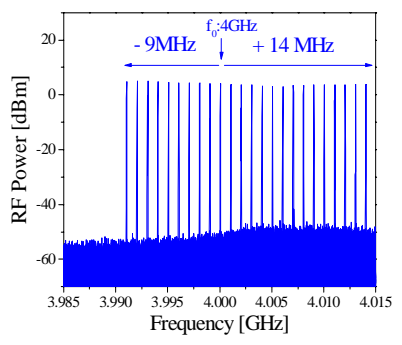

(a)

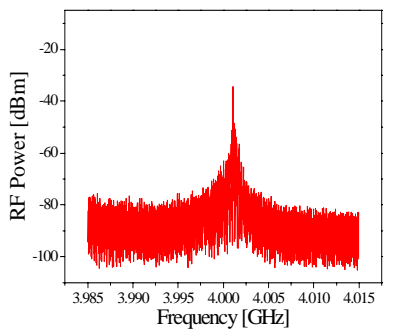

(d)

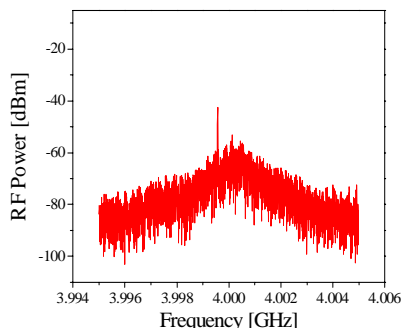

(b)

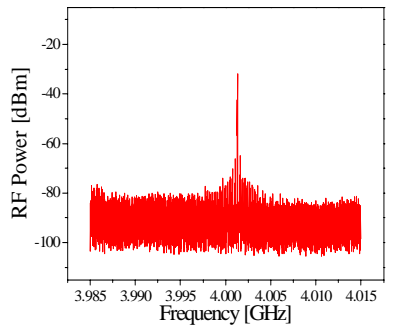

(e)

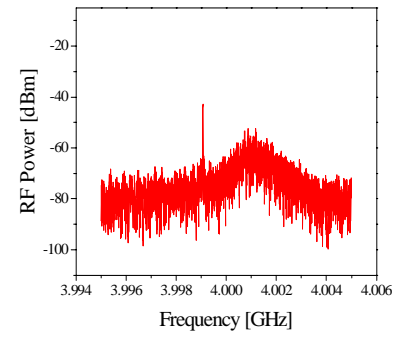

(c)

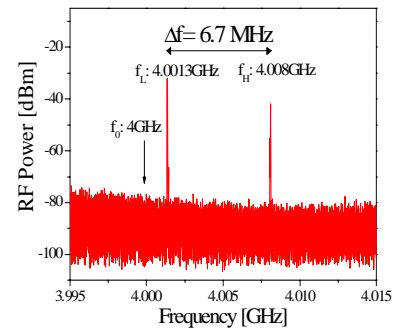

(f)

Fig. 7. RF spectra. (a) the detuned master laser, (b) (f) the interband optical pulse injected slave laser oscillating via ES transition. The cavity frequency offset of the slave laser with respect to the master laser is (b) $-500 \mathrm{kHz}$, (c) $-1 \mathrm{MHz}$, (d) $+1 \mathrm{MHz}$, (e) $+1.2 \mathrm{MHz}$ and (f) above $1.3 \mathrm{MHz}$.

We examined the locking dynamics of the slave laser as the repetition rate of the master laser is changed within the detuning range. First, as the injection rate is reduced from the nominal repetition rate of the slave laser, the slave laser does not lock to the interband optical pulse injection. The average repetition frequency of the slave laser after the interband optical pulse injection is shifted to higher rates rather than be locked to the master laser as shown in Figs. 7(b) and 7(c). Second, at higher injection rates than the nominal repetition rate of the slave laser, the repetition rate of the slave laser follows the master laser. As the repetition rate of interband optical pulse increases, the phase and amplitude noise decreases and the slave laser becomes locked to the master laser, whose cavity frequency varies from the $4.0013 \mathrm{GHz}$ to $4.008 \mathrm{GHz}$. A large locking bandwidth of $6.7 \mathrm{MHz}$ is achieved with an estimated $1 \mathrm{~mW}$ injection power as shown in Fig. 7(f) which shows the upper and lower limit of the recovered clock signal. We should note here that the locking of the interband injected slave laser is determined not only by the optical pulse train on DSS but also by the sideband noise suppression of over $30 \mathrm{~dB}$ from the fundamental RF tone. Even though we observed optical pulses on the DSS while the master laser was detuned above $4.009 \mathrm{GHZ}$, the sideband noise increased due to the large repetition rate offset between the master and slave laser. So the sideband noise reduction from the fundamental tone was less than $30 \mathrm{~dB}$ in the RF spectrum. 


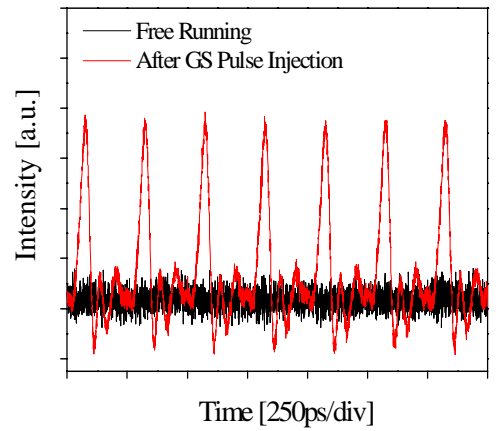

(a)

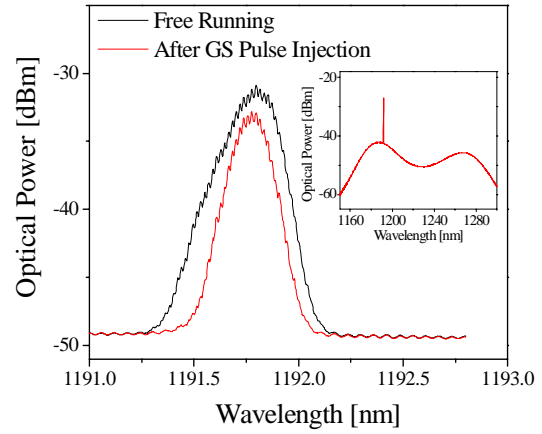

(b)

Fig. 8. (a). optical pulse train and (b) optical spectrum of the slave laser oscillating via ES transition before and after injection of GS optical pulse train of $4.004 \mathrm{GHz}$.

Figure 8 shows the optical pulse train and optical spectrum of the locked slave laser by an interband optical pulse injection at a repetition rate of $4.004 \mathrm{GHz}$. We also measure the locking bandwidth of the interband optical pulse injected slave laser oscillating via the ES transition in terms of the injected power and cavity frequency offset. The locking bandwidth is plotted in Fig. 9.

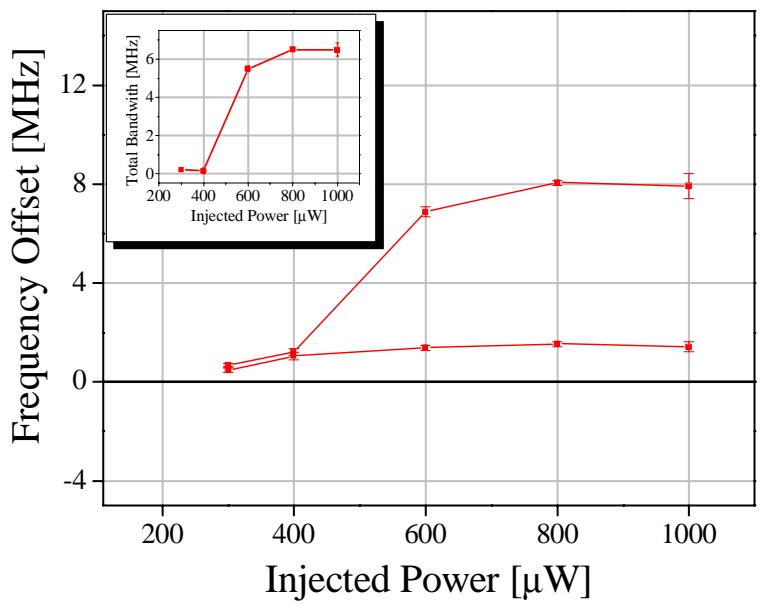

Fig. 9. Locking bandwidth of the slave laser oscillating via ES transition when GS optical pulse train is injected.

\section{Conclusion.}

In conclusion, we experimentally demonstrate that the slave laser oscillating via GS or ES transition can be locked through injection of optical pulses generated via the opposite transition bands, i.e. ES or GS transition. The locking bandwidths of an interband optical pulse injected laser were measured. First, when an ES optical pulse was injected to a slave laser oscillating via GS transition, the slave laser showed an asymmetric locking bandwidth and the slave laser was locked to a much higher injection rate. The maximum locking 
bandwidth of $500 \mathrm{kHz}$ was measured with an estimated $1 \mathrm{~mW}$ injection power. Second, when GS optical pulses were injected to the slave laser oscillating via ES transition, the locking of the slave laser was not achieved even though both the master and slave lasers run at the same repetition rate. The slave laser only becomes locked at higher injection rates than the nominal repetition rate of the slave laser. A maximum locking bandwidth of $6.7 \mathrm{MHz}$ was measured with an estimated $1 \mathrm{~mW}$ injection.

This interband optical pulse injection locking is achieved by interplay between the gain and absorption dynamics within the slave laser. These results represent to our knowledge the first observation of interband optical pulse injection locking in mode-locked semiconductor lasers.

\section{Acknowledgment}

We acknowledge support from DARPA under the University Photonics Research Center Program. 